\title{
La poética sentimental de Michel de Certeau
}

\author{
Eugenio Enrique CORTÉS RAMÍREZ \\ Universidad de Castilla - La Mancha \\ Departamento de Filología Moderna \\ eugenioe.cortes@uclm.es
}

Recibido: $31 / 10 / 2011$

Aceptado: 30/01/2012

\begin{abstract}
Resumen
La creación de una poética de la vida cotidiana ha sido una aspiración que fue lograda por Michel de Certeau. Pero no hay poética popular sin poética sentimental. Desde la perspectiva de la teoría cultural, la aportación de Certeau a los Estudios Culturales consiste en haber sentado las bases del sentimiento popular a través de una estética de la vida cotidiana, en contraposición a la visión de Henri Lefevbre, quien establece los prolegómenos de ese comportamiento popular en función de una teoría filosófica de la humanidad. Todo ese empirismo positivo fue heredado y utilizado por Michel de Certeau como base de una poética sentimental. Ahora les invitamos a descubrirlo.
\end{abstract}

Palabras clave: Michel de Certeau, Estudios Culturales Comparados, Teoría Cultural, Estética de la Vida Cotidiana, Poética Sentimental, Henri Lefevbre, Michel Foucault.

\section{La poétique du sentiment de Michel de Certeau}

\begin{abstract}
Résumé
L'article vise à éclairer les fondements pour la création d'une poétique de la vie quotidienne telle que conçue par Michel de Certeau. Mais il n'y a pas de sens populaire sans l'existence d'une poétique des sentiments. En ce qui concerne la théorie culturelle, la contribution de Certeau aux études culturelles a le mérite de poser les fondements du sentiment populaire à travers une esthétique de la vie quotidienne. Au contraire, Henri Lefebvre établit les prolégomènes de ce comportement populaire en se basant sur une théorie philosophique de l'humanité. Tout cet empirisme positif a été hérité et utilisé par Michel de Certeau comme le substrat sur lequel une poétique des sentiments peut être créé. Maintenant, nous vous invitons à la découvrir.
\end{abstract}

Mots clés: Michel de Certeau, études culturelles comparés, théorie culturelle, esthétique de la vie quotidienne, la poétique des sentiments, Henri Lefebvre, Michel Foucault.

\section{Michel de Certeau's Poetics of Sentiment}

\begin{abstract}
Michel de Certeau created a poetics of everyday life; there is no popular poetics without a poetics of sentiments. From the perspective of cultural theory, Certeau's contribution to Cultural Studies has laid the foundations of popular sentiment through the aesthetics of everyday life. Certeau opposed the views of Henri Lefebvre, who had set the prolegomenon of this popular behavior,, as based on a philosophical theory of humankind. All this positivist empiricism was inherited and used by Michel de Certeau as a basis for a poetics of sentiments, which is explored by this article.
\end{abstract}


Key words: Michel de Certeau, comparative cultural studies, cultural theory, aesthetics of everyday life, sentimental poetry, Henri Lefebvre, Michel Foucault.

\section{Referencia normalizada}

Cortés Ramírez, E. E., (2012) “La poética sentimental de Michel de Certeau”. Thélème, Vol 27. 105115 .

Ce que je souhaite vraiment établir est une science de la singularité; c'est-à-dire, une science du rapport qui lie des poursuites journalières aux circonstances particulières.

Michel de Certeau

Nunca podríamos haber hablado de la creación de una Poética Sentimental sin haber tenido en cuenta un antes y un después de la aparición de dos obras fundamentales en los Estudios Culturales franceses. Estas dos obras son Critique de la vie quotidienne (1947) del pensador francés de formación marxista Henri Lefebvre (1901-1991) y L'invention du quotidien ${ }^{1}$ (1974) del pensador jesuita Michel de Certeau (1925-1986).

Bien es cierto que sin la obra de Lefebvre, Certeau nunca podría haber contribuido con su imprescindible aportación. Pero esta primera apreciación nos conduce a establecer una comparativa entre ambas obras. Para Lefebvre, la vida cotidiana procede de la filosofía y no puede ser entendida sin ella. Para la filosofía, lo cotidiano designa lo que no es filosófico. Y lo que no es filosófico nace de la filosofía mediante el materialismo histórico. Como marxista, Lefebvre parte del materialismo histórico como mayéutica para revelar que la vida cotidiana nace del mundo científico que busca conocer el mundo real. Así, pues, para Lefebvre, el centro de la vida cotidiana es ocupado por el individuo y por la praxis concreta. Describir y analizar lo cotidiano nos conduce a mostrar su dualidad, su decadencia y fecundidad (Lefebvre, 1968: 22).

Por una parte, tanto el estilo como el pensamiento de Lefebvre están sustentados por una arquitectura crítica que ha sido generada por el tratamiento de la vida cotidiana como condición de alienación. Por otra, y en cambio, Certeau es seguidor de una tradición intelectual que parte del psicoanálisis hasta derivar en el postestructuralismo y en la postmodernidad, aunque este rasgo no le diferencia de

${ }^{1}$ Esta obra se divide en dos tomos y en tres autores: Michel de Certeau, L'invention du quotidien.1. Arts de faire, Gallimard, Paris, 1974; Luce Giard et Pierre Mayol, L'invention du quotidien.2. Habiter, cuisiner, UGE, Paris, 1980 (he consultado la edition de 1984). 
manera sustancial de la obra de Lefebvre. Quizás la diferencia más sustancial, está localizada en el plano sensible. Para Certeau la vida cotidiana surge del individuo como productor de sentidos. Por esta razón, lo cotidiano es psicológico y estético, fruto del conocimiento sensible. La producción de sentidos nace del desarrollo de la experiencia tanto física como espiritual que va más allá de la razón para referirse a una categoría más amplia de conocimiento. Se trata de seguir ese proceso meditativo del sentido donde la vida cotidiana produce una cierta disposición sentimental, que se convertiría en punto de partida del pensamiento cotidiano. Pero ese sentimiento necesita salir fuera de sí mismo y hacerse carne en una realidad ya sea vivida (la calle, la música, la resistencia, etc.) o inventada. Ese sentimiento, no obstante, ha necesitado de un estímulo externo como, por ejemplo, su atracción por la mística quietista del padre Jean Joseph Surin (1600-1665) para convertirse en inspiración (De Certeau, 1982: 89). En ese momento, el sentimiento dota de sentido a la intuición, y la intuición recoge el sentimiento. Así, pues, intuición, sentimiento e inspiración se producen de manera simultánea en una primera situación del discurso.

Una vez que el sentimiento ya ha forjado la idea, es necesario darle forma. Es aquí donde podríamos empezar a hablar de una poética del sentimiento, porque una vez que el sentimiento ha forjado la idea, lo necesario será darle una forma adecuada, bien en forma de imagen, bien en forma de escritura.

En cuanto a la escritura, Michel de Certeau concede una predominancia sobre la lectura. Porque los argumentos de la escritura son una ecléctica variedad de ejemplos y perspectivas teóricas que marcan una incuestionable fe en el potencial que ofrece cada nuevo discurso. Tal vez sea este el problema central al que se enfrenta Certeau. La vida cotidiana se oculta. Para tratar de asistir a ella, se exige algo así como un rito de fe. Quizás sea también el más sincero intento de aproximación a la vida cotidiana a través de los materiales de lo cotidiano. En caso de aproximación mediante el rito de la fe, se debe a que fe y creencia son las características de los tipos de conocimientos que circulan en la vida cotidiana. Si su teoría es mítica y sus argumentos están sometidos al esquema de lo cotidiano, se debe a que lo cotidiano puede ser pensado como el modelo de esta manera. Si para Certeau la escritura puede ser descrita como un proceso sugestivo y evocador, entonces la vida cotidiana también puede verse como textura de evocaciones que apuntan hacia una esfera sensorial registrable de manera inconmensurable mediante imágenes y palabras. En Certeau podemos encontrar un estilo propio para escribir sobre el día a día que está en armonía con su pensamiento.

Michel de Certeau era un sacerdote jesuita, además de miembro de la renombrada École Freudienne que dirigía el no menos afamado psicoanalista Jacques Lacan. También era un místico erudito comprometido en la modernidad de la historiografía crítica. Su compromiso profesional con aquello que él denominaba como escritura plural estaba reflejado en su perfil de etnógrafo de la vida cotidiana. Para Michel de Certeau, la escritura representa la imagen de un pensador itinerante. Su itinerario profesional estuvo marcado por la constante metáfora del viaje hacia el pensamiento y hacia aquellos lugares donde trabajó, como son Francia, Brasil, California, Argen- 
tina y Chile. El tema del viaje como movimiento activo a través del tiempo que camina hacia el espacio, compone la materialidad de la vida cotidiana para Certeau. Cuando leemos o caminamos, surge una sinfonía en el espacio y en el tiempo de todas aquellas actividades que están presentes en este juego. Viajar implica una mutación que altera no sólo al viajero, sino también a los espacios donde se produce el viaje. Viajar supone un encuentro con el otro, con las culturas, con la diferencia. El camino emprendido es una metáfora tan válida para Certeau como lo es la vida cotidiana. Es una opción por los asuntos pendientes, que se transforman en lugar de ser. En la escritura no hay un sistema acabado, una estructura que no puede ser superpuesta a lo cotidiano para producir territorios limpios y registrados en la cartografía del universo. Para Certeau, trabajar en lo cotidiano supone vivir una gran aventura. Y el camino que esta aventura emprende para teorizar la vida cotidiana, está trazado mediante una simbiosis entre el autor y su obra. Su ejemplo más claro son los dos volúmenes de L'invention du quotidien. En conjunto, representan la vida cotidiana como monumento teórico y empírico en ambas dimensiones. El primer volumen refleja al autor en sí mismo y, aunque bañado de ejemplos, se articula una aproximación teórica a la vida cotidiana. El segundo volumen está compuesto por los trabajos de Pierre Mayol y Luce Giard, combinado con ensayos breves del propio Certeau, donde ofrece una participación más sostenida en la práctica de la vida cotidiana. Para Certeau, su trabajo sólo debe ser tenido en consideración en conjunto con la labor de sus colegas. Los dos volúmenes fueron el resultado de un proyecto de investigación comenzado en 1974 y terminado en 1978, dirigido por el propio Certeau, bajo los auspicios de la prestigiosa Délégation Générale à la Recherche Scientifique et Technique. Esta circunstancia institucional puede parecer irónica a la hora de generar un proyecto que pueda revelar la diferencia entre el espacio táctico, puesto en funcionamiento sobre los más débiles, mediante el dominio estratégico que ha sido diseñado a través de poderosos proyectos políticos, económicos, científicos y racionales. Pero para Certeau, la teoría y la práctica que son necesarias para prestar atención a la vida cotidiana sirven como punto de partida en el proceso de investigación para L'invention du quotidien. Se trata de una ruptura histórica que inicia esta aventura.

El discurso de Certeau está situado junto al de otros intelectuales, que surgieron como partes integrantes de nueva cultura proclamada a raíz de de los sucesos de Mayo de 1968 (Rigby, 1991: 152). Para Certeau, la vuelta hacia el estudio de la vida cotidiana no se trata tan solo de la búsqueda de nuevos textos culturales para interpretarla y valorarla. Se trata también de un intento de definir la investigación sobre la forma de cómo poner en práctica la vida cotidiana. Según Certeau, la cultura popular, que surge en el seno de la vida cotidiana, pone en evidencia aquellas formas de utilización de los productos que nos han sido impuestos por un orden económico dominante (De Certeau et al., 1984: XIII). La vida cotidiana es el escenario de uso dentro de un sistema que, lejos de ser el suyo propio, ha sido construido para la propagación de otros (17). Lo que caracteriza a la vida cotidiana es la creatividad que surge de esta situación. No sólo para hacer ver al ser humano mediante esta creatividad, sino también, y fundamentalmente, esta creatividad nace 
para hacer con el ser humano actos de aprendizaje, de asimilación, y de reiteración. En otras palabras, estamos hablando de una poética de la vida cotidiana. Porque la vida cotidiana, para sobrevivir día tras día, tiene que desarrollar una capacidad de invención. A modo de ejemplo, puede ser comprobado cómo a través de aquellos materiales utilizados en las manualidades, la vida cotidiana potencia y valora la supuesta creatividad e ingeniosidad del bricolage. Entonces, el proceso creativo añade una nueva acepción a su significado, donde la creatividad es el acto de recombinación y la reutilización de materiales heterogéneos (49).

Sin embargo, estas asimilaciones no sólo son los productos de la voluntad de las personas o de sus acciones, sino que también son los productos de una cultura vista como heterogénea, de la cultura en plural. La heterogeneidad de la cultura se afirma en sí, no sólo a través de la actividad hija de la invención que nace de aquellas yuxtaposiciones hechas por la gente, sino a través de la obstinada insistencia del cuerpo, de los recuerdos de la infancia y de la historia cultural. Esta "resistencia de lo cotidiano", en palabras de Certeau, es una resistencia nacida de la diferencia, de la alteridad. Y por alteridad comprendemos aquellos obreros que están en contradicción con las máquinas que realizan su labor; aquellas tradiciones que se diferencian de quienes las promueven; aquellas visiones que son diferentes de los fundamentos que rigen el presente donde vivimos y cohabitamos. Certeau inicia esta partida hacia lo sensible en 1968, mediante su participación como respuesta a los acontecimientos de la primavera de París.

Porque un hecho es más importante que las reclamaciones que sugiere o incluso la impugnación a la que se ha sometido en estos términos antes que una multitud lo convirtiera en poético, aunque un tanto oculto. Pero estar oculto también implicará estar vivo. Esta vida silenciosa y silenciada se convirtió en discurso. Y este discurso explotó en el seno de aquellas relaciones que fomentó tanto con la fuerza de la alegría como con la pompa de la gravedad. Porque las categorías nacidas en lo cotidiano causan la destrucción de imprevistos y fomentan inexplicables lazos de solidaridad (De Certeau, 1974: 13). Escrito en el verano de 1968 como una declaración política, la descripción de los acontecimientos de Mayo comienza a condicionar algunos de los términos que aparecerán continuamente en sus investigaciones sobre la cultura popular y acerca de la vida cotidiana como son la creatividad de la asimilación, la poética de la experiencia, y el estilo de la vida cotidiana. Por supuesto, ese momento de la cultura emergente mediante la captación de la expresión podría ser visto como la sublimación de la vida cotidiana. Es, por tanto, cuando Certeau propone observar e investigar la invención poética en un plano más cercano a la cultura cotidiana. Para Certeau, "la vida cotidiana ya es extraordinaria, como un carnaval exuberante e incesante" (117). En este punto podemos decir que Certeau se despega de la obra de Lefevbre cuando reconsidera la vida cotidiana como una superación potencial de los límites impuestos por la norma. Pero se despega de ella para poder sobrevivir. Por esta razón, sería preciso aclarar aquellas posibles similitudes y diferencias entre ambos. Cuando Lefevbre escribe su Critique, nos descubre la vida cotidiana surgida en el seno de la modernidad capitalista donde se caracteriza por su "falta de estil" y por su "prosaico proceder". En sociedades anteriores, 
como la inca, la azteca, en Grecia o en Roma, todos los detalles que no son otra cosa que gestos, palabras, herramientas, utensilios, trajes, entre otros, todas tienen en común la impronta de un estilo donde nada había sido convertido todavía en prosaico, ni siquiera lo cotidiano, donde la prosa y la poesía de la vida siguen idénticos. Nuestra propia vida cotidiana está definida por la búsqueda de un estilo que elude obstinadamente hasta que, de manera inexorable e irreversible, lo invade todo, incluso la literatura, el arte y los objetos. Y en todos ellos, la poesía de existencia ha sido desalojada (Lefebvre, 1968: 29). Esta situación es, para Lefebvre, la colonización de la vida cotidiana por la prosa. La prosa es la impronta de la modernidad, caracterizada por una alienación del universo que ha sido penetrado. No sólo del lugar de trabajo, sino también de la vida cotidiana, el medio más importante. Los medios para superar esta condición están proporcionados por la vida cotidiana, pero nunca bajo aquella disciplina que la propia vida ha aceptado como ortodoxa. Tan sólo estarían proporcionados por aquellos métodos que la vida cotidiana considera como heterodoxos, y como consecuencia, alienados.

Por su parte, Certeau articula en su Invention una cuestión que podría poner en peligro la certeza de dicho análisis. Bien es cierto que, por una parte, esta disciplina ortodoxa de la vida cotidiana está en todas partes y ha sido aceptada de manera cada vez más clara y más amplia por la sociedad actual. Pero, por otra, es más urgente afirmar cómo toda esta sociedad se resiste a ser reducida a esta disciplina que ha sido organizada por unos sistemas disfrazados de procedimientos de carácter popular y que no lo son. Y estos procedimientos constituyen un estilo cuya puesta en funcionamiento supone una evasión de la vida cotidiana con el fin de silenciar aquellos procesos que organizan el establecimiento del orden socioeconómico imperante (De Certeau et al., 1984: XIV). Sin embargo, lo peor es que este estilo está disfrazado de resistencia al constante intento de colonización a la que la vida cotidiana está sometida. Este es el peligro que esta ambigüedad encierra en su contenido.

La posición de Lefebvre, donde defiende una versión matizada de la vida cotidiana, sostiene la idea de transformación cultural. Este concepto entra en contraposición con la necesidad de reproducción cultural, defendida por Michel Foucault, quien esta vez se suma a los postulados de Michel de Certeau para hacer hincapié claramente en la amenaza, tanto inmediata como futura, que supone la predominación de la lógica capitalista en la vida cotidiana. Esta profecía de Foucault (1975) donde el hombre occidental parece estar abocado inexorablemente al economicis$\mathrm{mo}^{2}$, parece tener eco, por desgracia, en las crisis inmobiliarias que asolan el pano-

\footnotetext{
${ }^{2}$ Economicismo es un término utilizado para criticar el reduccionismo económico, que es la reducción de todos los hechos sociales a dimensiones económicas. También es usado para criticar la economía como una ideología, donde la oferta y la demanda son los únicos factores importantes en toma de decisiones, y literalmente sobrepasa o permite ignorar todos los otros factores. Esta corriente
} 
rama económico global de 2011. Certeau y Foucault defienden la imposibilidad de la plena colonización de la vida cotidiana por el sistema, mediante la continuación de la resistencia permanente a la lógica temporal de capitalismo democrático, y el estallido de la estética del heterogéneo como primer paso hacia una poética del sentimiento. La diferencia entre de Certeau y Lefebvre también se muestra en los resultados políticos que se derivan de la vida cotidiana. Para Lefebvre, el resultado de este análisis es una praxis revolucionaria que obtendrá provecho de estos momentos de sensibilidad, momentos donde germinan las semillas de una sensibilidad que posibilita una realidad diferente cada día. Para Certeau, la instrumentalización de la política tiene que estar en suspenso por necesidad.

Según Certeau, la primera lección que hemos aprendido del Mayo del 1968 es el fracaso de una poética popular basada en el mensaje oficial de reforma política y organizada, que pretende generar una reconquista de la expresión por parte de la autoridad y pone de manifiesto la decadencia real y verdadera de sus posibilidades (De Certeau, 1974: 29-31). La escritura de los sauvages que caracterizó las protestas de 1968 y los años siguientes, ofrecen un tipo de movimiento bajo la forma de cultura, porque sus participantes ya no pueden demandar sus postulados sociopolíticos dentro de los marcos tradicionales de referencia (112). Para el poder establecido, dichas solicitudes son irrupciones en la vida cotidiana, irrupciones que no tienen ninguna oportunidad de ser escuchadas por la tajante política tradicional.

Desde Mayo de 1968, la política tradicional fue precisamente el enemigo más oficial que no tenía nada que decir en respuesta a las pintadas que habían surgido sobre los muros de París. Hasta Mayo de 1968, la derecha y la izquierda, Gaullisme et Gauchisme, se habían convertido en uno (Rigby, 1991: 96). La escritura popular brotada en los muros hablaba de exigencias de diferenciación, de aceptación tanto de la realidad como de la diversidad cultural. Estas consignas resonaron con una serie de actividades culturales y referencias políticas que recordaron al surrealismo con un toque de anarquismo. Si los deseos fueron recapturados por los reformistas, o por los marxistas resurgidos de su propia autocrítica, Certeau se mantuvo preocupado por el eco del deseo de analizar ese qué es ese algo que la sociedad demanda a

oportunista surgió en la filosofía de la socialdemocracia rusa a finales del siglo XIX y comienzos del $\mathrm{XX}$. Los denominados economicistas procuraban circunscribir los objetivos del movimiento obrero a la mera lucha económica, con medidas como mejora de las condiciones de trabajo, elevación de salarios, entre otras. Según esta filosofía, la lucha política era tarea de la burguesía liberal. Los economicistas negaban el papel del partido, de la clase obrera y su teoría revolucionaria, exaltaban la espontaneidad del movimiento obrero. Como variedad del revisionismo, el economicismo servía de medio conductor de la influencia burguesa sobr el proletariado. La difusión del economicismo obstaculizaba la creación de un partido proletario centralizado. El periódico leninista Iskra (La chispa) llevó a cabo una labor ingente para poner al desnudo la inconsistencia del economicismo. La derrota ideológica de dicha corriente fue conseguida por Lenin mediante su obra ¿Qué hacer?, publicada en 1902. 
gritos. Pensar que las diferencias entre Lefebvre y Certeau tan sólo tienen lugar en términos de resultados políticos, supone perder algunas de las más productivas similitudes que comparten. Lo cierto es que hubo grandes puntos de convergencia entre ambos autores. Ambos comparten la visión de la vida cotidiana como una praxis. Ambos conciben la vida cotidiana como algo fenomenológico y sensual, como un reino estético que requiere la atención de una poética sentimental sobre el estilo de vida. En este punto, Lefebvre aprecia que el problema que plantea el estilo, es que no puede ser puesto en práctica plenamente según las condiciones actuales. Esta lista de puntos comunes comienza a dar vida a infinidad de conexiones, no sólo entre Lefebvre y de Certeau, sino también en toda la gama de escritos y prácticas que hasta ahora hemos venido examinando.

\section{Elementos para la creación de una poética sentimental}

Para Certeau, la poética postmoderna, surgida a finales del Siglo XX, está realizada por el hombre sin atributos. Para la creación de tal poética, y aunque pudiera parecer paradójico, tres cualidades de este héroe sin atributos deberían ser tenidas en cuenta. Primero, podríamos hablar de su sometimiento a las reglas del consumo. Porque el consumo realizado es siempre activo y creativo. Nunca está sometido a la forma del producto. Segundo, habría que constatar su extrema habilidad en el uso de ciertas argucias para vencer la presencia anónima del poder. En otras palabras, sabe cómo vencer al fuerte. Tercero, estar dotado de cierta sensibilidad para recrear redes de intersubjetividad paralelas a los grandes poderes. En este caso, Certeau difiere de Foucault, quien considera que el hombre ordinario manifiesta una subjetividad constituida por y desde el poder. Sin embargo, Certeau resalta la capacidad de resistencia constante del hombre común contra el poder.

La simbiosis de estas tres cualidades genera la producción de una poética de la vida cotidiana. Tal poética surge de las prácticas de la vida cotidiana y permite estas prácticas a ser visible y audible, con el fin de marcar su distancia de los tradicionales marcos de referencia socio-políticos. Para Certeau, significa la generación de una poética sutil dotada de elementos tácticos suficientes para permitir la creación de un arte de hacer cada día. Gracias a este arte de hacer, la vida cotidiana aparece como un medio de resistencia. Sin embargo, esta resistencia no es sinónimo de oposición. En la concepción de Certeau, el término resistencia se acerca más a la utilización del término en el dominio del psicoanálisis y de la electrónica. Es aquello que obstaculiza y que disipa el flujo de energía de la dominación. Es, por tanto, lo que se resiste a la representación.

En sus escritos sobre la vida cotidiana, resistencia es una actividad que nació de la inercia como resultado de la invención como formas de dar crédito a la existencia cotidiana. Por una parte, comprende fenómenos que están en desarrollo de manera permanente y de un modo lento. Son las latencias, aquellos retrasos que se amontonan en la amplia dimensión de las mentalidades, y de sus consecuentes ritos sociales como medio de lucha contra la opacidad, elemento que impide el desarrollo del 
proceso creativo. Por otra parte, también se producen irrupciones, desviaciones, interferencias. Es decir, todos estos márgenes que retan el ingenio de las generaciones futuras cuando tratan de cultivar la cultura (De Certeau, 1974: 137-138). La gruesa amplitud de la opaca e intensa vida camina de la mano con aquellas desviaciones que generan la invención en este panorama de la vida cotidiana. Si bien esto podría presentar cierta similitud con el trabajo de Raymond Williams, y su énfasis en el concepto de residual y emergente, donde hace diferencia entre las culturas reales y positivas que se diferencian de las culturas dominantes (Williams, 1958: 121-127). Esta diferencia no se encuentra simplemente en la continuación de las prácticas culturales ni tampoco en los valores que ya no están en boga como, por ejemplo, las tradiciones rurales. Así, pues, tanto Certeau como su equipo de investigación desean registrar una densidad cultural en torno a las prácticas y los objetos que evocan aquello que podría ser pensado como un bien cultural inconsciente o imaginario.

Este objetivo se logrará si las prácticas cotidianas, los modos de funcionamiento o arts de faire, ya no se limitan a aparecer como el fondo oscuro de la actividad social. En cambio, sí aparecen como un conjunto de cuestiones teóricas, de métodos, categorías, y de perspectivas que penetran en esta oscuridad, para hacer posible la articulación entre todos ellos (De Certeau, 1984: XI). La condición de posibilidad para un proyecto de este tipo está basada en la invención de una poética, una poiesis, una capacidad de invención denominada la poética de lo cotidiano. Esta poética deberá ser comprendida como una investigación sobre aquellas formas que toma la vida cotidiana y como una actividad surgida de la capacidad poética del lenguaje y de la vida. Certeau nos recuerda que la etimología del término poética viene del griego poiein, que significa crear, inventar, generar (De Certeau, 1984: 205).

El deseo de desubicar este análisis fuera de los marcos sociopolíticos tradicionales de referencia se ha cumplido por su regreso. El lenguaje de la ley y la transgresión, del poder y de la resistencia, ha obstaculizado la investigación de las posibilidades abiertas por Certeau. Fundamentalmente, cuando Certeau sienta las bases para la creación de una poética sentimental, desempeña un complicado juego. Al tratar de escapar de la reducción de la posible bipolarización del idioma, su pensamiento escrito no hace más que emplear una serie de términos binarios. El primer volumen de L'invention du quotidien está sostenido por medio de la orquestación de términos binarios: Por ejemplo, el consumo versus la producción. La lectura frente a la escritura. Las tácticas frente a las estrategias. La palabra hablada frente a la escrita. Lo que hace Certeau mediante este uso de términos binarios es desafiar las estructuras del pensamiento binario. Semánticamente contrapuestas, términos como estrategias y tácticas se niegan a ser simples antagonistas en un debate sobre el poder y la resistencia. En su lugar conceden la oportunidad a la diferenciación. Por ejemplo, no sólo los términos producción y consumo son causa y efecto el uno del otro, sino que cada uno proporciona al otro la complementariedad esencial para ser definidos mutuamente.

En realidad, una producción de carácter racionalizado, expansionista, centralizada, y espectacular se enfrenta a una clase totalmente diferente de producción, el 
llamado consumo, que se caracteriza por su fragmentación como resultado de circunstancias, su carácter furtivo y clandestino, su incansable, pero tranquila actividad. En pocas palabras, debido a su cierta invisibilidad cuasi plena no tiene apenas capacidad de poder mostrar sus propios productos. Pero sí domina un arte de hacer capaz de utilizar aquellos productos que le fueron impuestos (De Certeau, 1984: 31). Estas asociaciones productivas han tenido repercusiones cruciales para el empleo de otros términos binarios. Por ejemplo, en el uso de los términos lectura y escritura, Certeau minimiza y disuelve las diferencias entre ellos: Tenemos que dejar de pensar que una diferencia cualitativa existe entre los actos de lectura y escritura. El primero es un silencio creativo, surgido de lo que el lector hace con el texto y la segunda está basado en la misma creatividad, pero en la producción de un nuevo texto, de forma explícita. Siempre presente en la lectura, la actividad cultural sólo encuentra una variante y una prórroga por escrito. Desde la escritura hacia la lectura y viceversa, ninguna la línea diferencial separa la pasividad de la actividad, excepto la línea que distingue entre diferentes modos sociales para dejar una brecha abierta entre una práctica y su puesta en funcionamiento bajo una forma determinada (De Certeau, 1974: 145).

La única diferencia que puede mantenerse entre la lectura y la escritura es el acto social peculiar de valorar la actividad que es más visible por estar escrita. Cuando esto ha sido puesto en evidencia, tanto la lectura como la escritura, en las prácticas tienen que ser diferenciados. Pero no en función de su visibilidad, sino por sus formas de funcionamiento. Pero ahora esa diferenciación ya no sería capaz de operar dentro de las convenciones designadas por los términos lectura y escritura. Esta táctica de utilizar términos binarios no está exenta de problemas. Para Certeau, el éxito mediante ellos puede parecer irregular. Su utilización con éxito parece depender de una lógica de relación que no sólo debe ser referida a circunstancias de carácter práctico. Sino que es también un uso que requiere la adquisición de una densidad metafórica, más allá de ser verificables y actividades de dominio determinado, como con la oposición en contra de la oralidad de la escritura, o de la lectura frente a la escritura. Si el comentario de Certeau ha sido alterado por el deseo de desvincular estos desvíos para volver a establecer oposiciones binarias, es, en parte, porque el trabajo puede ser considerado como una vuelta a activar una división entre el poder absoluto y su falta del mismo. Según Certeau, los textos tienen la misma densidad porosa que podemos encontrar en la propia vida cotidiana. Porque son relativamente abiertos a diferentes lecturas, que son capaces de suministrar el material para muy diversos tipos de argumentos.

A la hora de escribir este trabajo, la atención puesta en su escritura no ha sido otra que el reconocimiento del trabajo de Certeau como las bases para establecer una poética donde la vida cotidiana estaría condenada a surgir y, por tanto, a vivir. Y esta poética de la vida cotidiana la llamaremos de ahora en adelante Poética Sentimental. 


\section{REFERENCIAS BIBLIOGRÁFICAS}

Ahearne, J., (1995) Michel de Certeau: Interpretation and its Other. Cambridge, Polity.

Buchanan, I., (2000) Michel de Certeau, Cultural Theorist. London, SAGE Publications Ltd.

De Certeau, M., (1966) Correspondance de Jean Joseph Surin. Paris, Desclée de Brouwer.

De Certeau, M., (1970) La possession de Loudun. Paris, Julliard, Coll. Archives.

De Certeau, M., (1974) L'invention du quotidien 1. Arts de faire. Paris, Gallimard.

De Certeau, M., (1982) La Fable Mystique. Paris, Gallimard, Coll. Bibliothèque des Idées.

De Certeau, M.; Giard, L. \& P. Mayol, (1984) L'invention du quotidien 2. Habiter, cuisiner. $1^{\mathrm{e}}$ édition 1980, Paris, UGE.

Dosse, F., (2002) Michel de Certeau. Le marcheur blessé. Paris, La Découverte.

Foucault, M., (1975) Surveillir et punir. Paris, Gallimard, Coll. Bibliothèque des Histories.

Hoggart, R., (1957) The Uses of Literacy. London, Chatto \& Windus.

Joyce, J., (1922) Ulysses. (Edición española de José María Valverde, Barcelona, Bruguera, 1975), Paris, Shakespeare and Co.

Lefebvre, H., (1958) Critique de la vie quotidienne. Vol. I: Introduction. Paris, L'Arche.

Lefebvre, H., (1958) Critique de la vie quotidienne. Vol. II: Fondements d'une sociologie de la quotidienneté. Paris, L'Arche.

Lefebvre, H., (1958) Critique de la vie quotidienne. Vol. III: De la modenité au modernisme (Pour une métaphilosophie du quotidien). Paris, L'Arche.

Lefebvre, H., (1968) La vie quotidienne dans le monde moderne. Paris, Gallimard, Coll. Bibliothèque des Idées.

Schutz, A., (1962) Collected Papers I: The Problem of Social Reality. Edited by M.A. Natanson and H.L. Breda, Dordrecht, Martinus Nijhoff Publishers.

Rigby, B., (1991) Popular \& Modern Culture in France. A Study of Cultural Discourse. New York, Routledge.

Williams, R., (1958) Culture \& Society. (New edition in 1963, with a new introduction, New York, Columbia University Press), Chatto \& Windus, London. 